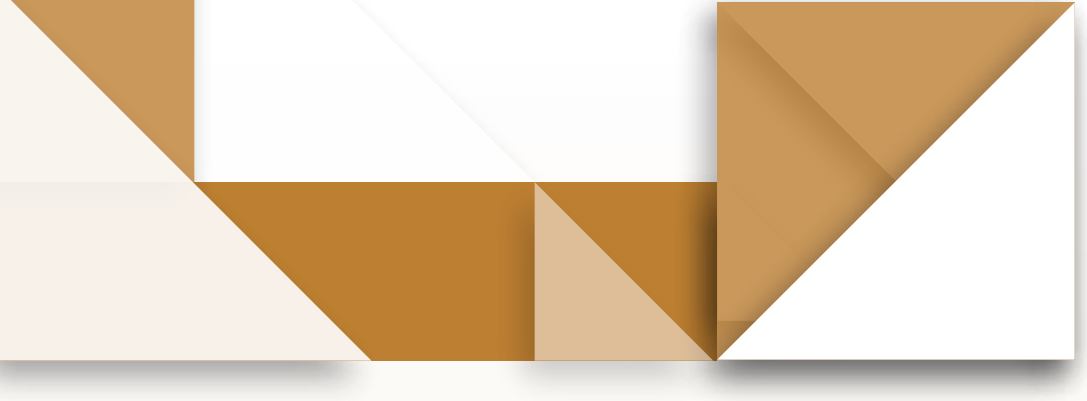

\title{
Más allá de la profesionalización: los quehaceres de la educación
}

\section{- Beyond Professionalization: the Tasks of Education}
- Além da Profissionalização: as tarefas da educação

\section{Resumen}

Este artículo de reflexión muestra la necesidad de pensar, más allá de la profesionalización, la formación de profesores como un continuo desde una práctica performativa, es decir, desde la experiencia que se piensa y afirma en los quehaceres pedagógicos y didácticos que cotidianamente se construyen y reconstruyen en el aula. Asimismo, es una reflexión desde la política pública sobre el problema estructural del acceso a la educación como una práctica de exclusión, que, entre otras, refleja las relaciones de inequidad, desigualdad e injusticia social. El artículo analiza en un primer momento el problema de la desfinanciación de la universidad pública en Colombia; enseguida, se refiere al binomio formación-profesionalización; en tercer lugar, se refiere al desequilibrio entre la oferta y la demanda, y finalmente analiza la función de la política educativa en la formación docente. Los aspectos analizados permiten concluir que el bienestar de las personas está influenciado por la calidad de la educación que reciben por lo que la formación incide de manera directa en el ejercicio profesional. Por otra parte, se concluye sobre la oferta y la demanda que existe una imperiosa necesidad de cualificar los perfiles de acuerdo con los contextos, lo cual implica que la política pública educativa contemple de manera efectiva la cualificación del profesor en los niveles de maestría y doctorado, y que la retribución por estos estudios se vea reflejada en los salarios que perciben. Finalmente, el modelo de formación basado en la confianza de las capacidades de los sujetos de generar conocimiento profesional especializado mediante la práctica marca nuevos retos tanto para esos sujetos como para las instituciones de educación superior.

Palabras clave

Formación docente; profesionalización; financiación; política pública; educación superior; oferta y demanda

\section{Myriam A. Zapata Jiménez*}

Doctora en Estudios Latinoamericanos por la Universidad Nacional Autónoma de México (unam). Docente investigadora del Doctorado de Educación y Sociedad de la Facultad de Ciencias de la Educación de la Universidad de La Salle, Bogotá, Colombia.

Correo electrónico: myzapata@unisalle.edu.co 


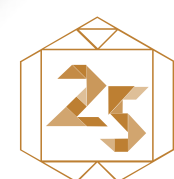

Años

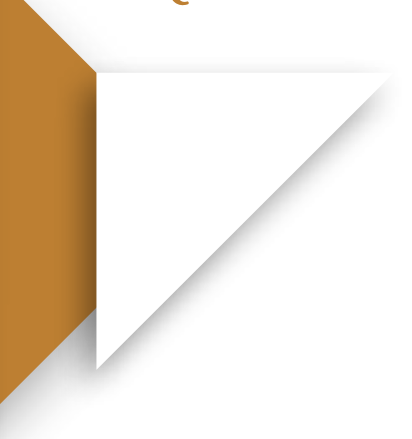

\section{Abstract}

This reflection article shows the need to think, beyond professionalization, the training of teachers as a continuum from a performative practice, that is, from the experience that is thought and affirmed in the pedagogical and didactic tasks that are built and rebuild daily in the classroom. It is also a reflection from public policy on the structural problem of access to education as a practice of exclusion, which, among others, reflects the relations of inequity, inequality and social injustice. At first, the article analyzes the problem of the lack of financing of the public university in Colombia; next, it refers to the binomial training-professionalization; thirdly, it refers to the imbalance between supply and demand, and finally it analyzes the role of educational policy in teacher training. The aspects analyzed allow us to conclude that the well being of people is influenced by the quality of education they receive, so that training directly affects their professional practice. On the other hand, it is concluded about the supply and demand that there is an urgent need to qualify the profiles according to the contexts, which implies that the educational public policy effectively contemplates the qualification of the teacher at the masters and doctorate levels, and that the remuneration for these studies be reflected in the wages they receive. Finally, the training model based on the confidence of the subjects' abilities to generate specialized professional knowledge through practice marks new challenges both for those subjects and for higher education institutions.

\section{Keywords}

Teacher training; professionalization; financing; public policy; higher education; supply and demand

\section{Resumo}

Este artigo de reflexão mostra a necessidade de pensar, além da profissionalização, a formação de professores como um contínuo a partir de uma prática performativa, ou seja, a partir da experiência pensada e afirmada nas tarefas pedagógicas e didáticas que são construídas e reconstruídas diariamente na sala de aula. É também uma reflexão das políticas públicas sobre o problema estrutural do acesso à educação como prática de exclusão, que, entre outros, reflete as relações de iniquidade, desigualdade e injustiça social. $\bigcirc$ artigo analisa, inicialmente, o problema da definição da universidade pública na Colômbia; a seguir, refere-se à binomial formação-profissionalização; terceiro, refere-se ao desequilíbrio entre oferta e demanda e, finalmente, analisa o papel da política educacional na formação de professores. Os aspectos analisados permitem concluir que o bem-estar das pessoas é influenciado pela qualidade da educação que recebe, de forma que o treinamento afeta diretamente sua prática profissional. Por outro lado, conclui-se quanto à oferta e à demanda que há uma necessidade urgente de qualificar os perfis de acordo com os contextos, o que implica que a política pública educacional contempla efetivamente a qualificação do professor nos níveis de mestrado e doutorado e que a remuneração desses estudos seja refletida nos salários que eles recebem. Por fim, o modelo de treinamento baseado na confiança das habilidades dos sujeitos em gerar conhecimento profissional especializado por meio da prática marca novos desafios, tanto para os sujeitos quanto para as instituições de ensino superior.

Palavras-chave

Formação de professores; profissionalização; financiamento; políticas públicas; ensino superior; oferta e demanda 


\section{Introducción}

Quiero comenzar refiriéndome a algunos aspectos que inciden de manera directa sobre la formación docente. Podemos decir que las desigualdades sociales han llevado a que el acceso a la educación sea un privilegio y no un derecho, lo que desde la acepción social de la sustentabilidad contradice el principio que se relaciona con la democracia, los derechos humanos, la paz y la equidad (Organización de las Naciones Unidas para la Educación, la Ciencia y la Cultura, Unesco, 1997). De acuerdo con la Comisión Económica para América Latina y el Caribe (Cepal):

La exclusión del ámbito educativo en todos los niveles no solo es una vulneración grave de los derechos de los niños, niñas, adolescentes y jóvenes, sino que además limita sus posibilidades de participación en otras esferas de la sociedad en igualdad de condiciones. El acceso a la educación es aún más restringido para aquellos que viven en zonas rurales y en situación de pobreza. $(2017$, p. 75).

Si bien es cierto se ha avanzado en indicadores de cobertura de educación en América Latina, la brecha entre pobreza y riqueza continúa marcando la diferencia en cuanto a la calidad y el acceso a la educación en todos su niveles y modalidades, así como a la empleabilidad. Un diagnostico general nos muestra algunas causas críticas que se relacionan directamente con cuestiones estructurales: una política centrada en la normatividad; la homogeneización curricular, didáctica y evaluativa, y el desconocimiento de los enfoques diferenciales; la cobertura sin calidad y la centralización de las políticas públicas educativas; la urbanización de la educación rural, situada y diferencial; la carencia de una política pública efectiva para la educación rural, que atienda los problemas de cobertura con calidad e infraestructura, $y$, finalmente el debilitamiento de la autonomía y la institucionalidad.

De acuerdo con Federico Mayor de Zaragoza:

Promover el desarrollo sostenible, cuyas estrechas relaciones con la democracia y con la paz cada vez son más patentes, es una de las tareas fundamentales de nuestra época, y para realizarla con éxito es vital la educación en todas sus modalidades. La Unesco cree en la educación como fuerza del futuro — que no puede ser sino un futuro sostenible- - y está empeñada en obtener el máximo fruto posible de sus esfuerzos y en multiplicar sus actividades en pro del desarrollo y del despliegue de esta fuerza a favor de la paz y de la mejora de los seres humanos. (Citado en Unesco, 1997, p. 3).

De acuerdo con este ideal, no podemos dejar de preguntarnos cuál ha sido el papel de la política educativa en la formación profesional, y hasta qué punto la formación y la cualificación de profesores se convierte en una fuente de desarrollo o, como piensan algunos, en un obstáculo. ¿̇Cuál tendría que ser la respuesta de las universidades públicas y privadas frente a la actual crisis financiera? $Y \dot{z}$ desde dónde comenzar a pensar las instituciones de educación en todos sus niveles, no solo en lo que se refiere a las nuevas competencias y fortalezas profesionales, si no a las fuentes de financiación?

\section{La financiación de las universidades públicas en Colombia}

Uno de los temas de la actual crisis de la universidad pública se relaciona con el financiamiento, que es del $10 \%$ desde la Ley 30 de 1992. Se espera que en el Plan de Desarrollo 2019 se incremente dicho presupuesto, debido a que los ingresos crecen al ritmo del índice 
de precios al consumidor (IPC), mientras que los gastos aumentan cerca de 4 puntos por encima del IPC:

Entre 2005 y 2016, los recursos públicos que recibió la Universidad de Antioquia disminuyeron $26 \%$, mientras que los recursos propios aumentaron $88 \%$. Para 2018, el déficit financiero de la institución es de 181.528 millones de pesos. Ese dinero alcanzaría para que ningún estudiante de pregrado o posgrado pagara su matrícula durante seis años. (Múnera, 2018, p. 4).

El problema de la desfinanciación de la educación superior pública resulta más complejo cuando se realizan señalamientos sobre la poca efectividad de los programas o se desprestigia la identidad de profesores y estudiantes por el ejercicio de la protesta social; cuando se impiden la libre expresión y el pensamiento crítico. Múnera' señala que el financiamiento dual del Sistema Universitario Estatal (SUE) estipulado en la Ley 30 de 1992 generó un impacto negativo en la autonomía universitaria. Dicha financiación es, por una parte, fuente pública (artículos 86 y 87), y por otra, "fuente privada de bolsillo y crédito", que se ve reflejada en la reducción del gasto de aportes estatales para las instituciones educativas públicas, que pasaron de transferir 84 de cada 100 pesos a 49. Es así como la inversión estatal se redujo del 84 al $49 \%$.

Mientras en 1993 el Estado transfería ochenta y cuatro de cada cien pesos que gastaban las universidades públicas, en la actualidad aporta cuarenta y nueve de cada cien. Con el paso del tiempo, las universidades pasaron asumir [sic] la mitad de los gastos con los recursos que son capaces de generar. Eso se tradujo en matrículas más altas, en la venta de bienes y servicios, en el endeudamiento y en el deterioro de las condiciones laborales de profesores y trabajadores. 32 universidades públicas con déficits de 15 billones. ("Pública la recibimos, pública la entregaremos", 2018, p. 2).

Dentro de las demandas de las universidades públicas está la reforma a los artículos 86 y 87 de la Ley 30 de 1992, de tal forma que se incrementen en 8 puntos los recursos asignados a partir del IPC o se determine un porcentaje de incremento a partir del PIB, para garantizar el funcionamiento de las instituciones públicas de educación superior de acuerdo con sus funciones misionales. Los siguientes escenarios de cobertura de la tabla 1 muestran las disparidades en términos de la matrícula para la educación superior, que incluye técnico y tecnológico, veamos:

Encuentro Nacional de Profesores Universitarios "Crisis de la Universidad pública; mitos y realidades". Asociación de Profesores de la Universidad de Antioquia (Asoprudea). Universidad de Antioquia, Medellín, 18 de septiembre del 2018 . 
Tabla 1. Escenarios de cobertura 2034

\begin{tabular}{lccc}
\hline \multicolumn{1}{c}{ Indicadores } & Lista de base (2014) & Escenarios (2034) \\
\hline Tasa de cobertura bruta educación superior & $50 \%$ & Intermedio & Ideal \\
\hline & Pregrado & $70 \%$ & 84 \\
\hline Matrícula pregrado (total) & 2.178 .444 & 3.087 .094 & 3.900 .627 \\
\hline Matrícula universitaria & 1.370 .682 & 1.234 .838 & 1.365 .219 \\
\hline Total de nuevos cupos creados en pregrado & $(\ldots)$ & 908.650 & 1.722 .183 \\
\hline Participación de la matricula universitaria & $63 \%$ & $40 \%$ & $35 \%$ \\
\hline Participación de la matrícula técnica y tecnológica & $37 \%$ & $60 \%$ & $65 \%$ \\
\hline
\end{tabular}

Fuente: cálculos de Mora y Múnera con base en CESU, Acuerdo por lo Superior 2034. Propuesta de política pública para la excelencia de la educación superior en Colombia en el escenario de la paz. Bogotá: CESU, 2014, p. 25.

Un aspecto que determina la financiación se relaciona con el sistema de indicadores de capacidad y resultados del modelo del artículo 87 con vigencia al 2013 que se refieren a la formación (índice de resultados de formación, Irfor). En la propuesta metodológica para la distribución de recursos se señalan, entre otros, los siguientes indicadores:

- Número ponderado de programas académicos de pregrado y posgrado ofrecidos por la institución.

- Número ponderado de matriculados en primer curso por niveles de formación y metodologías de enseñanza en pregrado.

- Número ponderado de matriculados por niveles de formación y metodologías de enseñanza en pregrado y posgrado.

- Número ponderado de graduados en el nivel de formación de pregrado y posgrado por metodologías de enseñanza y áreas de conocimiento.

- Número ponderado de estudiantes de la Universidad que obtuvieron un puntaje mayor al quintil superior en las pruebas saber pro [sic].
- Número de estudiantes con resultados B2 en el examen de inglés en la prueba Saber Pro.

- Número de graduados en el año anterior vinculados laboralmente al sector formal.

- Número de estudiantes en programas de movilización en universidades internacionales con las que se tiene convenio.

- Número de estudiantes extranjeros matriculados en cualquier nivel educativo de Instituciones de Educación Superior -IES- internacionales con las que se tiene convenio. (Ministerio de Educación Nacional, MEN, 2013, p. 4).

Para la vigencia 2016 los objetivos se concentraron en apoyar los esquemas de planeación y ejecución de los procesos académicos, de investigación y administrativos de las IES, con base en los resultados de los componentes del modelo. Del mismo modo:

- Establecer una distribución de los recursos que sea consecuente con el objetivo del Ministerio de Educación Nacional de mejorar la cobertura 
con calidad y los demás objetivos contemplados en el Plan Nacional de Desarrollo.

- Generar incentivos para que las IEs públicas registren la información a tiempo y con calidad en el SNIES.

- Diseñar una metodología de distribución de recursos que valore el desempeño de las IES como organizaciones eficientes y competitivas dentro de las exigencias de la comunidad académica internacional.

- Apoyar al Ministerio de Educación Nacional y el Estado en general, así como a las IES, en la autorregulación del sector de la educación superior y la focalización de sus estrategias de fomento. (MEN, 2016, p. 3).

Si bien es cierto que los índices de calidad se han mantenido, en cumplimiento de los criterios de evaluación y acreditación, la mayoría de ellos resultan inviables presupuestalmente. Respecto de los indicadores, estos se quedan cortos a la hora de decidir por la excelencia de la calidad, como lo muestran las clasificaciones internacionales, que ubican a la Universidad Nacional y a la de Antioquia entre las mejores de América Latina.

\section{La formación y la profesionalización}

La profesionalización y la formación docente también ofrecen matices que requieren diferenciarse. Además de los problemas de acceso por financiación, la complejidad de modalidades y el contexto global son aspectos útiles de considerar en el momento en que se elaboran los programas. La profesionalización es un proyecto que crece y se difunde desde el espacio estatal donde se gestan e implantan las reformas educativas contemporáneas. Gran parte de su fuerza se origina externamente en la medida en que es un elemento básico del programa de modernización educativa impulsada por organismos internacionales como la Unesco, el Banco Mundial y el Banco Interamericano de Desarrollo (BID).

Entendemos por profesionalización un proceso continuo de perfeccionamiento personal y laboral en que intervienen varios elementos comunes de la ocupación laboral y que se vincula a una determinada práctica profesional. Desde este punto de vista, profesionalización y desarrollo profesional son sinónimos. En el marco del proceso de reconstrucción del rol profesional, una de las tareas sustantivas es desarrollar en los estudiantes las capacidades que les permitan reflexionar sobre la propia práctica, independientemente de que estén en el nivel de posgrado. Para Fullan y Hargreaves (Citados en Pérez, 1993), la expresión desarrollo profesional tiene un sentido amplio y debe "incluir cualquier actividad o proceso que intenta mejorar destrezas, actitudes, comprensión o actuación en roles actuales o futuros". Este va más allá de la sola información: implica adaptarse a los cambios con el propósito de modificar las prácticas educativas; se preocupa por las necesidades personales, profesionales y organizativas. 
Una visión más dinámica y flexible torna el concepto de profesionalidad más sensible y permeable a los cambios sociales y acepta la coexistencia de concepciones divergentes. Esta posición implica la visión del profesional como un práctico-reflexivo, que analiza su práctica, que utiliza el conocimiento de una manera flexible, dependiendo de la naturaleza de las situaciones. La reflexión-en-la-acción representa la posición en la que el profesional puede improvisar soluciones a nuevos problemas, reflexionar sobre su propio conocimiento y su práctica, y valorar lo que funciona o no en diferentes situaciones. La reflexión como forma de indagación se considera la vía por la que los prácticos pueden problematizar o cuestionar su propio quehacer. Se deriva, así, el perfil de un profesional reflexivo y crítico abierto al cambio, capaz de analizar su práctica para transformarla.

En la relación formación/profesionalización ambas cuestiones se encuentran atravesadas por paradigmas en los que la validez del conocimiento mantiene la lógica de la superioridad del conocimiento universal de las ciencias naturales sobre el conocimiento de lo singular de las ciencias sociales. Cuando se habla de formación del profesorado, los enfoques formativos adquieren relevancia no solo desde la perspectiva curricular y didáctica, sino desde las epistemes que determinan las competencias y la identidad profesional. En este sentido, los enfoques formativos en ciencias naturales privilegian la investigación experimental de acuerdo con la cual, por ejemplo, el egresado de Química de la Universidad Pedagógica Nacional desarrollará competencias para

Diseñar, experimentar y validar un currículo para la formación de profesores de química, que desde sus elementos constitutivos posibilite la formación de un profesor de química caracterizado en cuanto al saber químico por los presupuestos científicos y epistemológicos, que lo fundamentan y que se sustentan en el marco teórico. (Perfil del egresado, s.f).

Por el contrario, el perfil de un profesor egresado del programa de Filosofía de la Universidad Nacional Abierta y a Distancia (UNAD) será: "Diseñador y gestor de proyectos de carácter interdisciplinario que promueven el aprendizaje y apropiación de los saberes pedagógicos, filosóficos y humanísticos en diferentes niveles de la educación" (Perfil del egresado, s.f.), o el de la Universidad Santo Tomás: "Formar intelectuales capaces, mediante la investigación e interpretación del patrimonio filosófico universal y latinoamericano, de profundizar, actualizar y proyectar la tradición humanística de Colombia" (Egresos, s.f.).

Otro elemento que se constituye en un indicador de la formación se relaciona con los enfoques epistemológicos. De acuerdo con un estudio desarrollado por Gallego, Pérez, Torres de Gallego y Torres sobre la formación inicial y continua de maestros en ciencias en Colombia:

En general, ninguno de los programas plantea la formación inicial de profesores, en términos del cambio en las concepciones epistemológicas, pedagógicas y didácticas con las cuales ingresan los estudiantes a dichos programas. No hay en ellos referencias a las concepciones alternativas. Tampoco hay discursos en torno a la evaluación. Igualmente, en lo relacionado con la historia interna de las teorías o modelos de las ciencias objeto de formación [sic]. (2004, p. 2).

Estas prácticas y saberes marcan diferencias entre el enfoque formativo y el quehacer educativo, lo cual se refleja en temas y problemas como el de la sustentabilidad. El artículo 109 de la Ley 115 de 1994 describe los fines de la formación del educador respecto de la calidad científica y ética; la teoría y práctica pedagógica como parte

\author{
AXIS

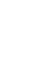


del saber del educador; el fortalecimiento de la investigación pedagógica y el saber específico; y, finalmente, la formación respecto de los diferentes niveles y prestación del servicio. Por eso llama la atención la ausencia de enfoques alternativos o su insipiencia en la formación docente.

Estos elementos son necesarios para reflexionar sobre la relación entre la formación y la práctica pedagógica en sus diferentes niveles y escenarios. En este sentido, respecto de la función docente, el artículo 4 del Decreto 1278 del 2002 señala, además de las actividades de actualización y perfeccionamiento, otras que se refieren a procesos sistemáticos de enseñanza-aprendizaje, además del diagnóstico, la planificación, la ejecución y la evaluación de procesos y resultados, y otras actividades formativas, culturales y deportivas en el marco de los proyectos educativos institucionales. Por otra parte, la función docente contempla actividades curriculares no lectivas, el servicio de orientación estudiantil, la atención a la comunidad, en especial de los padres de familia de los educandos.

Otro aspecto de particular interés se relaciona con la formación de posgrado. Una mirada al crecimiento de la matrícula en posgrado en las IES durante la década de los noventa en América Latina permite explicarla desde la necesidad de cualificación de la inserción laboral. Este crecimiento del sector generó crecientes demandas de formación que coadyuvara a la práctica profesional de los egresados. No obstante, en muchos casos esta expansión no se ha visto acompañada por un planeamiento estratégico en relación con el desarrollo productivo, cultural y educativo.

En este contexto, las propuestas formativas para el posgrado obedecieron a demandas coyunturales originadas en las nuevas definiciones de la política educativa, así como en la necesidad de adecuarse a los cambios culturales globales y económicos, por lo que configuraron una oferta fragmentada y poco sistemática. Es así como la universidad se convierte en un espacio de reproducción del mundo capitalista, guiado por la rentabilidad y competitividad del mercado global. Desde esta perspectiva, las universidades tienen como objetivo de formación:

Producir mercancías "cognitivas" adaptadas al mercado o formar trabajadores cualificados, también para el mercado. De ahí que la figura de la universidad-empresa sea inseparable de la constitución de un "mercado del conocimiento" y de la configuración subjetiva del trabajador cognitivo propio de este tipo de capitalismo. (Edu-Factory y Universidad Nómada, 2010, p. 8).

De ahí que en este contexto surjan cambios significativos, como los contemplados en la Ley 30 de 1992, que han llevado cada vez más a la mercantilización y el lucro de la educación. Hemos asistido a la bancarización del Icetex y al sistema policivo de Colciencias patrocinado por el Banco Mundial, quien dictamina, clasifica y descalifica lo que no entra en su mercado global cognitivo. Y qué no pensar sobre las desigualdades e inequidades en materia educativa, a pesar de las acreditaciones de alta calidad de las instituciones, que ya no 
otorgan becas, sino préstamos, lo que para el caso de la educación pública resulta aún más lesivo, con un financiamiento entre el uno y el tres por ciento adicional al IPC en el 2019. A propósito de la inversión de la empresa privada en proyectos de investigación varias universidades se han preguntado hasta qué punto ese interés privado puede chocar con su misión y con su verdadera función.

Mientras que no se resuelvan estas desigualdades de formación en el pregrado, concebir un programa de posgrado implica partir de una lectura común de este escenario educativo, para prever acciones. Más allá de las viejas preocupaciones por la relación de los educadores con el conocimiento, su producción, circulación y distribución social exige la atención de los procesos sustantivos de la formación, ante las transformaciones actuales y el lugar de la diversificación cultural, el acceso equitativo, la autonomía institucional y el fortalecimiento de la democracia a partir de las apuestas humanizantes desde las que nos invita Martha Nussbaum a pensar la educación, desarticulada de los marcos empresariales, sin fines de lucro.

En el contexto de estas perspectivas y prioridades, la formación del posgrado para los profesionales de la educación o aquellos provenientes de otras áreas que desarrollan actividades en el ámbito educativo cobra particular relevancia en el proceso de construir una mejor escuela y de contribuir al desarrollo humano.

De acuerdo con la misión de la educación superior propuesta por la Unesco, esta se debe enfocar principalmente en mantener, incrementar y difundir el conocimiento mediante la investigación, la creación intelectual, la enseñanza y difusión de actividades educativas en los diversos sectores sociales con miras a su desarrollo, crecimiento de la persona y de la sociedad: al ampliar el acceso, la educación superior debe tratar de alcanzar simultáneamente los objetivos de equidad, pertinencia y calidad. La equidad no es únicamente una cuestión de acceso - el objetivo debe ser la participación y conclusión con éxito de los estudios, al tiempo que la garantía del bienestar del alumno. Este empeño debe abarcar el adecuado apoyo económico y educativo para los estudiantes que proceden de comunidades pobres y marginadas (Unesco, 2009).

Siendo fieles a esta misión, las IES tienen el reto de generar formas de promoción y financiación de los estudios de posgrado.

En este sentido, los estudios de posgrado constituyen, por una parte, la oportunidad de actualizar, ampliar y profundizar los conocimientos culturales y profesionales; y por otra, un interés por iniciar y orientar la práctica profesional diversificada del posgrado al desarrollo humano y social. Es indispensable la formación en competencias no solo académicas, por lo que requiere un enfoque prioritario de profesionalización del quehacer cotidiano. Esto quiere decir, que desarrolle un modelo educativo tendiente a la formación de profesionales con aptitudes y competencias específicas, que le permitan interactuar en el ámbito laboral y disciplinario, así como responder a las exigencias permanentes de cambio de la sociedad global.

Desde la perspectiva de los repertorios sociales y culturales específicos, para hablar de una educación situada, la relación entre profesionalización y formación supone concebirla como una práctica interactiva, auto y performativa a lo largo de la vida profesional del docente. En el contexto de posgrado, se entiende la función de investigación como práctica sistemática de elaboración de conocimiento que permitirá avanzar en la profundización y comprensión de los fenómenos educativos, 
reconocer al profesional como investigador de su propia práctica y coadyuvar al mejoramiento de la educación y a la profundización de los niveles de profesionalización.

El modelo de profesionalización orientado a la indagación es una de las alternativas de mayor impacto en la conceptualización del desarrollo profesional del docente. Comprende el conjunto de actividades de reflexión e indagación que los docentes realizan, que pueden ser la investigación-acción participativa $u$ otro tipo de investigación realizada en el aula o en la escuela. Pensemos por ejemplo en los contextos de violencia y vulnerabilidad que vivimos en Colombia: la educación para la paz supone replantear desde estas dinámicas las formas de intervención y acción pedagógica. Este modelo de desarrollo tiene como objetivo propiciar hábitos de indagación; formar docentes autónomos, reflexivos, investigadores en la acción, científicos, que resuelvan problemas e indaguen acerca de su propia práctica.

Las bases de este modelo de formación las encontramos en las propuestas de Dewey, quien escribió que los profesores necesitan "una acción reflexiva". Otro de los impulsores de este modelo es Zeichner (1993), quien señala que desde hace más de cuarenta años se ha planteado el tema de "los profesores como investigadores sobre la acción", a partir de allí el autor se refiere a diferentes aspectos entre los cuales se encuentra la innovación, la autodirección y la observación participante. Uno de los elementos claves de este modelo es considerar la investigación como reflexión sobre la práctica, pues a través de ella se detecta y resuelven problemas.

En este sentido, la investigación académica generaría una práctica y un conocimiento profesional para las demandas y situaciones concretas de los entornos sociales. Ambas creencias se cuestionan cada vez más. Al mismo tiempo, quienes se dedican a la enseñanza han manifestado su preocupación por la falta de conexión existente entre la idea de conocimiento profesional que prevalece en los centros de formación y aquellas competencias que se les exigen a los prácticos en el terreno de la realidad institucional en busca del cambio o la solución de problemas.

Es necesario plantear al revés la cuestión de la relación entre la competencia en la práctica y el conocimiento profesional. No deberíamos empezar por preguntar cómo hacer un mejor uso del conocimiento científico, sino qué podemos aprender a partir de un detenido examen del arte, es decir, de la competencia por la que en realidad los profesionales son capaces de manejar las zonas indeterminadas de la práctica, independientemente de aquella otra competencia que se pueda relacionar con la racionalidad técnica.

Se debe replantear tanto la epistemología de la práctica como los supuestos pedagógicos para favorecer cambios institucionales, de modo que den cabida a una práctica reflexiva como un elemento en la preparación de sus profesionales. Un desarrollo formativo profesionalizante implica un concepto de epistemología 
de la práctica. Se puede tomar como fundamento lo que propone Schön (1983 y 1987) como alternativa a la racionalidad técnica y que permite analizar el proceso de formación. Dicha alternativa epistemológica de la práctica se apoya en la idea de la reflexión en la acción, más adecuada para entender ciertas profesiones que incluyen procesos artísticos intuitivos en la toma de decisiones, como son las vinculadas a la función educativa en general.

Por lo tanto, el objetivo de profesionalización se sustenta en el ejercicio de una práctica integral, mediante la cual desarrollar las competencias tendientes a la profesionalización del quehacer cotidiano, relativo a la recuperación y sistematización de la experiencia educativa.

\section{Desequilibrio entre la oferta y la demanda}

En la misión, las universidades se comprometen con la formación de profesionales comprometidos con la excelencia académica y fortalecidos en un ideal humanista global de respeto a la diversidad, comprometidos con la justicia y la renovación social. Además, fortalecer la conformación intelectual y profesional de cuerpos académicos y docentes de acuerdo con los requerimientos institucionales e instancias educativas del país. A partir de estos ideales, se proyecta que su visión debe tender hacia la consolidación como institución de educación superior de la más alta calidad.

Esto significa que la formación de profesores requiere poner sobre el tapete la apertura de los marcos desde los cuales se comprenden la formación y se concibe el currículo hacia apuestas transdisciplinares. Sin embargo, el desequilibrio entre la oferta y la demanda ha llevado a la ortodoxia de perfiles, que incide en la desprofesionalización y el desempleo, por lo que la educación superior hoy requiere reorientarse en función de competencias diversas, dada la complejidad y necesidades del mundo global. Un cuestionamiento al respecto tiene que ver con la corporativización y la manera como esta responde a las dinámicas globales de la transnacionalización de la educación en la formación y contratación de mano de obra calificada para la división internacional del trabajo. Instituciones como Unisánitas, Compensar, Colsubsidio, CET-EADS, así como la propia Universidad del Ministerio de Tecnologías de la Información y las Comunicaciones, MinTic, se postulan como el modelo que "sí atiende a las necesidades reales del país". Diana María Silva (vicerrectora académica de la Corporación de Educación Tecnológica Colsubsidio, CET) señala entre sus ventajas:

- La estructura modular permite la generación de rutas de formación que favorecen el paso de la educación no formal (cursos, diplomados) a la educación formal (carreras técnicas).

- Diseño currículos flexibles a la medida de las necesidades de la compañía.

- Selección de expertos en los temas, de acuerdo con experiencias reales dentro de los sectores productivos y orientados al desarrollo de competencias.

- Transferencia dinámica de un oficio y desarrollo de habilidades y competencias en interacción cotidiana.

- Horarios ajustados a las dinámicas del negocio.

- Formación pertinente para el desarrollo de competencias organizacionales y específicas de la organización.

- Utilización de la capacidad instalada de las empresas.

Si bien es cierto que la educación corporativa atiende a necesidades específicas del ámbito empresarial que, por supuesto, son válidas y obedecen a los intereses formativos

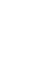


de cada persona, se convierten en la única posibilidad de cualificación y acceso a la educación. Por lo tanto, más allá de los debates sobre la profesionalización entre ciencias duras y blandas, la cuestión de la formación de profesores en la actualidad supone generar respuestas educativas situadas en el contexto específico de los colectivos y comunidades para potenciar sus saberes, sus discursos, sus quehaceres educativos, y fortalecer su carácter relacional con las dinámicas del mundo global. Qué enseñar, cómo y desde dónde son, entre otros, temas y problemas que nos corresponde explicar a la luz de la formación docente hoy.

En este sentido, la discusión sobre la superioridad de las ciencias resulta irrelevante, máxime cuando se habla de sociedades sustentables en un contexto de calentamiento global, hambrunas, migración forzada, xenofobia, y todas las fobias hacia la diversidad. El problema de la sustentabilidad no se resuelve solo en las aulas. ¿̇ué es sustentable en una sociedad líquida, en la que el fracking por la explotación minera está acabando con nuestros recursos? ¿De qué vale enseñar a los niños el cuidado del agua, de las plantas, de la biodiversidad, cuando no se realizan los esfuerzos reales y suficientes por parte de los Estados para su preservación?

\section{La función de la política en la formación docente}

La política pública educativa en América Latina se encuentra determinada por las políticas educativas internacionales de la Organización para la Cooperación y el Desarrollo Económicos (OCDE), así como por los planes de desarrollo de los gobiernos, quienes determinan los rumbos sociales y educativos de acuerdo con su programa. Es así como la Agenda 2030 de la Unesco propone un enfoque integral del desarrollo sostenible, indivisible, holístico, integral y sistémico. Sin embargo, los desbalances entre lo público y lo privado, entre lo rural y lo urbano mantienen las brechas de desigualdad e inequidad y de justicia social.

El marco normativo de la formación de educadores consagrado en el artículo 111 de la Ley 115 de 1994 señala la necesidad de formación permanente; la actualización y el mejoramiento profesional, así como el perfeccionamiento científico e investigativo de los educadores, especialmente en maestría y doctorado. Sin embargo, los estímulos son bajos considerando que en Colombia existían 161920 docentes oficiales en el 2018. La política educativa en el país es el gendarme de un Estado regulador de las dinámicas del capitalismo cognitivo. La fuga de cerebros o su utilización al referirnos a la cobertura y el acceso deja políticas cojas a la hora de su ejecución, en parte por la falta de sostenibilidad de la misma política atada a los gobiernos de turno.

Respecto de Ser Pilo Paga, existen posturas en pro y en contra sobre la terminación de este programa, que benefició a 39992 estudiantes, sin recursos, para el acceso a la educación privada. Creemos que no se puede frenar una medida que por lo menos garantizó que el 2 \% de la población accediera a dicha educación, por lo que el criterio de que es muy bajo el beneficio y muy alto el costo 
no tiene un asidero. También es cierto que con el presupuesto de 3,5 billones de pesos se podría pagar la matrícula de 450000 estudiantes en instituciones públicas y no privadas, como reclamaron varios estudiantes de la Universidad Nacional en el Encuentro Nacional de Estudiantes en marzo, septiembre y octubre del 2018 (Redacción Vivir, 21 de marzo del 2018). Ahora bien, la necesaria terminación de la formación de quienes iniciaron dichos programas, y de quienes ya no tendrán acceso a él, así como el endeudamiento mediante el Icetex para acceder a la educación superior forman parte de los retos por superar en el nuevo Plan de Desarrollo.

Estas brechas y desbalances marcan diferencias entre la formación técnica y la profesional, la rural y la urbana. Entre las limitaciones del sistema educativo rural se señala, entre otras,

Un sistema de educación superior que al no haber logrado desarrollarse como un conjunto de entidades investigativas ha devenido en un sistema profesionalizante en donde la formación en disciplinas ha sido desplazada por un sistema de baja calidad de formación en profesiones. (Echeverri, 1998, pp. 139-140).

Los índices sintéticos de calidad muestran resultados bajos respecto a los indicadores de logro en los repertorios rurales, en los que la mitad de la población rural dispersa en Colombia es analfabeta. Respecto a este tema, vale la pena señalar la importancia que tienen las Escuelas Normales Superiores (ENS) para el sector rural, ya que ofrecen desde el grado de preescolar hasta el grado $11 \mathrm{y}$, adicionalmente, cuentan con un Programa de Formación Complementaria ( $P F C$ ) para la preparación de docentes que atienden preescolar y básica primaria. En términos del fortalecimiento de la formación docente en el sector rural, el país tiene una deuda enorme, dado que en la mayoría de casos, estos profesores sólo acceden al ciclo de formación complementaria.

En el marco de los procesos de acreditación de 1997 y 2008, por lo menos el 50 \% de los programas de formación complementaria de las escuelas normales superiores desapareció al no alcanzar los indicadores de calidad. Esto llevó a un retroceso en los procesos de formación inicial de profesores rurales. Esta dificultad obedece principalmente a las inequidades territoriales respecto del acceso a la educación superior. Pese a ello, las ENS han cumplido un papel preponderante en la formación de profesores en los territorios más apartados de Colombia, con población dispersa y rural. Una descripción general de las ENS hecha por el MEN nos muestra que:

- El $6 \%$ de los docentes del país son normalistas (19.408) y el $69 \%$ de ellos se desempeña en zona rural.

- 96 escuelas normales superiores, de 137 que tiene Colombia, se encuentran ubicadas en municipios de menos de 100 mil habitantes.

- Las escuelas normales superiores desarrollan trabajo de extensión y apoyo a comunidades, como alfabetización, capacitación a madres comunitarias, educación rural y atención a grupos étnicos.

- Nueve departamentos, Arauca, Putumayo, Amazonas, Risaralda, Quindío, Casanare, Vichada, Caquetá y Vaupés, cuentan con una Escuela Normal Superior y en el caso de Vichada, Vaupés, Arauca y Putumayo es la principal alternativa para que los jóvenes bachilleres puedan formarse como docentes. 
- 3 departamentos, San Andrés, Guainía y Guaviare, no cuentan con escuela normal. (Las escuelas normales superiores y el Ministerio de Educación verifican las condiciones de calidad de los programas de formación complementaria, 2018).

Esta caracterización es un indicativo de la incidencia positiva que tienen las escuelas normales superiores en los territorios. En el Sexto Encuentro Nacional de Escuelas Normales Superiores, llevado a cabo en Bogotá el 6 de abril del 2018, se subrayó la necesidad de fortalecer la formación complementaria para el ejercicio profesional en los niveles de Educación Básica Secundaria y Media.

Otro aspecto por analizar es la economía de la educación, que - de acuerdo con una investigación en curso, dentro de la Línea de políticas públicas, calidad de la educación y territorio del Doctorado en Educación y Sociedad_- se refiere a la demanda y la oferta de educación y a la teoría del capital humano. Esta última alude a la relación entre los costos y los beneficios asociados a las decisiones de inversión en educación que hacen las personas. Por ejemplo, cuando alguien decide estudiar una carrera universitaria, incurre necesariamente en unos costos directos, tales como el costo de las matrículas, los materiales de estudio, los libros, así como el costo indirecto asociado a los ingresos laborales que se dejarían de percibir durante el tiempo de estudio. Por el lado de los ingresos, la persona debería evaluar el incremento en estos que va a recibir como consecuencia de una mayor productividad, que se supone está asociada a las habilidades y conocimientos desarrollados en su proceso educativo en la universidad. Los economistas llaman capital humano a ese conjunto de habilidades y conocimientos que las personas acumulan a lo largo de su proceso educativo, el cual —dicho sea de paso- debe tener el potencial de aumentar su productividad y, por ende, sus ingresos a lo largo de su vida laboral.

Por otro lado, se entiende como demanda de educación aquello que la sociedad requiere de su sistema educativo para formarse, al tiempo que por oferta educativa se entiende el conjunto de programas de formación que ofrecen las instituciones educativas a la sociedad. En este orden de ideas, la tabla 2 muestra las áreas de conocimiento con mayor demanda social de acuerdo con los intereses formativos y de ingreso laboral.

Desde la perspectiva de la economía de la educación, una persona que estudia Ciencias de la Educación y se desempeña como docente tiene una remuneración salarial de acuerdo con el grado del escalafón. Según datos suministrados por el MEN, la nómina del Estado a febrero del 2018 asciende a \$161 911 (51,32\%) educadores oficiales. El número de docentes en cada uno de los escalafones y la remuneración salarial vigente se muestran en la tabla 3. 
Tabla 2. Matrícula por área de conocimiento

\begin{tabular}{llccccccc}
\hline \multicolumn{1}{c}{ Área de conocimiento } & 2010 & 2011 & 2012 & 2013 & 2014 & 2015 & 2016 \\
\hline $1 \quad \begin{array}{l}\text { Economía, administración, } \\
\text { contaduría y afines }\end{array}$ & 525470 & 576550 & 602662 & 668635 & 721100 & 745067 & 781102 \\
\hline 2 & $\begin{array}{l}\text { Ingeniería, arquitectura, } \\
\text { urbanismo y afines }\end{array}$ & 475685 & 516520 & 538744 & 590598 & 622924 & 649333 & 670353 \\
\hline 3 & Ciencias sociales y humanas & 248046 & 274743 & 292550 & 321391 & 355456 & 378055 & 407085 \\
\hline 4 & Ciencias de la educación & 141391 & 170884 & 174914 & 179999 & 190424 & 192879 & 202013 \\
\hline 5 & Ciencias de la salud & 141239 & 155032 & 157113 & 161582 & 162592 & 161522 & 162879 \\
\hline 6 & Bellas artes & 66857 & 72005 & 74054 & 79642 & 79461 & 76681 & 78391 \\
\hline 7 & Matemáticas y ciencias naturales & 34552 & 35858 & 37951 & 40803 & 42478 & 44753 & 47201 \\
\hline 8 & Agronomía, veterinaria y afines & 40781 & 58100 & 51598 & 50241 & 46217 & 45260 & 45410 \\
\hline & TOTAL & 1674021 & 1859692 & 19295862092891 & 2220652 & 2293550 & 2394434 \\
\hline
\end{tabular}

Fuente: snies-men (2017)

Tabla 3. Número de docentes y remuneración salarial

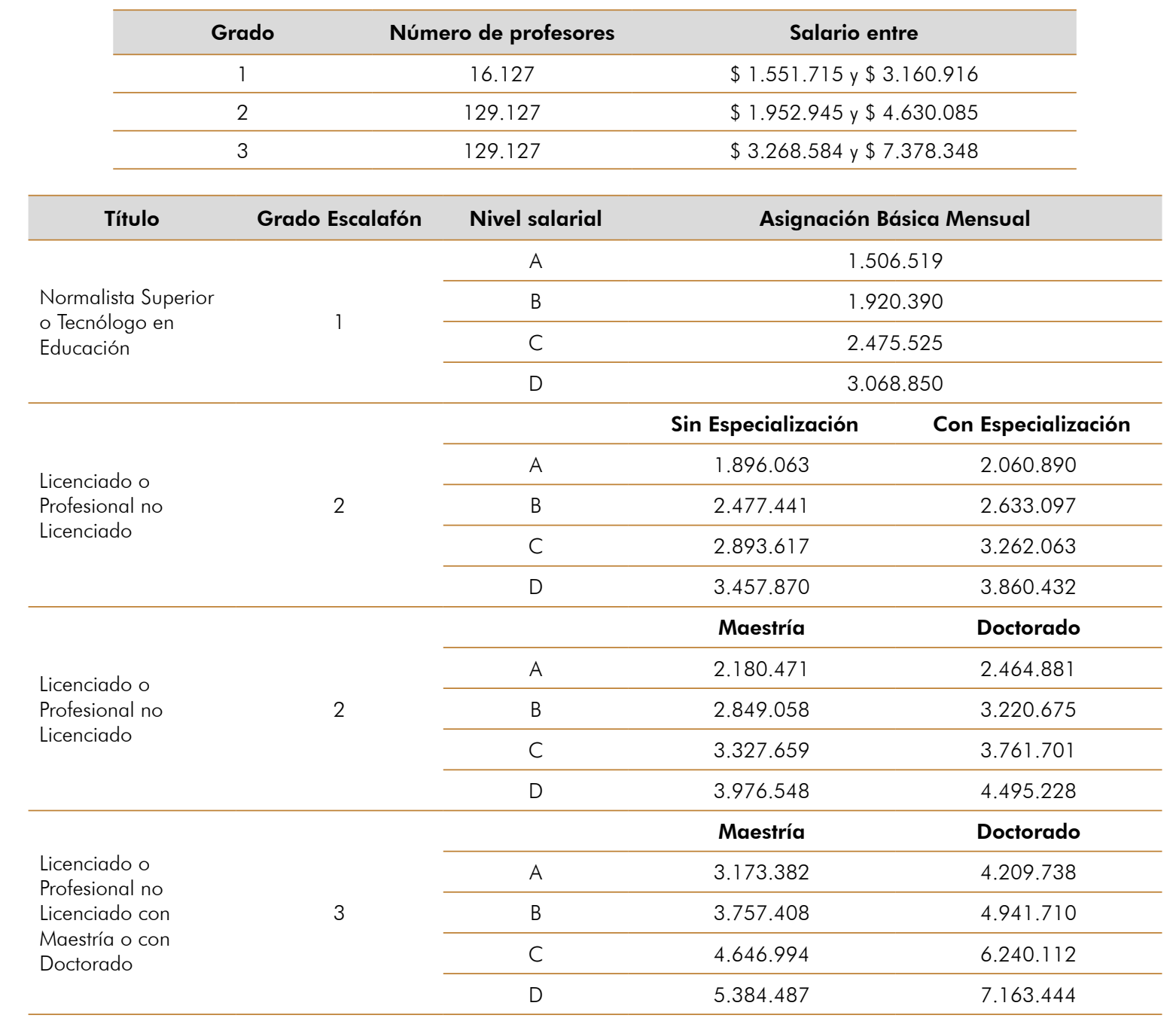

\begin{tabular}{|c|c|c|c|c|}
\hline \multicolumn{3}{|c|}{ Numero de proiesores } & \multicolumn{2}{|c|}{ Salario entre } \\
\hline \multicolumn{2}{|c|}{1} & 16.127 & \multicolumn{2}{|c|}{$\$ 1.551 .715$ y $\$ 3.160 .916$} \\
\hline \multicolumn{2}{|c|}{2} & 129.127 & \multicolumn{2}{|c|}{$\$ 1.952 .945$ y $\$ 4.630 .085$} \\
\hline \multicolumn{2}{|c|}{3} & 129.127 & \multicolumn{2}{|c|}{$\$ 3.268 .584$ y $\$ 7.378 .348$} \\
\hline Título & Grado Escalafón & Nivel salarial & \multicolumn{2}{|c|}{ Asignación Básica Mensual } \\
\hline \multirow{4}{*}{$\begin{array}{l}\text { Normalista Superior } \\
\text { o Tecnólogo en } \\
\text { Educación }\end{array}$} & \multirow{4}{*}{1} & A & \multicolumn{2}{|c|}{1.506 .519} \\
\hline & & B & \multicolumn{2}{|c|}{1.920 .390} \\
\hline & & C & \multicolumn{2}{|c|}{2.475 .525} \\
\hline & & $\mathrm{D}$ & \multicolumn{2}{|c|}{3.068 .850} \\
\hline \multirow{5}{*}{$\begin{array}{l}\text { Licenciado o } \\
\text { Profesional no } \\
\text { Licenciado }\end{array}$} & \multirow{5}{*}{2} & & Sin Especialización & Con Especialización \\
\hline & & A & 1.896 .063 & 2.060 .890 \\
\hline & & B & 2.477 .441 & 2.633 .097 \\
\hline & & C & 2.893 .617 & 3.262 .063 \\
\hline & & $\mathrm{D}$ & 3.457 .870 & 3.860 .432 \\
\hline \multirow{5}{*}{$\begin{array}{l}\text { Licenciado o } \\
\text { Profesional no } \\
\text { Licenciado }\end{array}$} & \multirow{5}{*}{2} & & Maestría & Doctorado \\
\hline & & $A$ & 2.180 .471 & 2.464 .881 \\
\hline & & B & 2.849 .058 & 3.220 .675 \\
\hline & & C & 3.327 .659 & 3.761 .701 \\
\hline & & $\mathrm{D}$ & 3.976 .548 & 4.495 .228 \\
\hline \multirow{5}{*}{$\begin{array}{l}\text { Licenciado o } \\
\text { Profesional no } \\
\text { Licenciado con } \\
\text { Maestría o con } \\
\text { Doctorado }\end{array}$} & \multirow{5}{*}{3} & & Maestría & Doctorado \\
\hline & & A & 3.173 .382 & 4.209 .738 \\
\hline & & B & 3.757 .408 & 4.941 .710 \\
\hline & & C & 4.646 .994 & 6.240 .112 \\
\hline & & $\mathrm{D}$ & 5.384 .487 & 7.163 .444 \\
\hline
\end{tabular}

Fuente: Decreto 316 de 2018. 
De lo anterior se desprende, de acuerdo con Isaza (2017), la pertinencia del enfoque de la economía de la educación en el contexto del Doctorado en Educación y Sociedad. No se trata únicamente de ver la educación como un mercado más en el cual se transan mercancías comunes y corrientes. Más bien se trata de estudiarla como un "bien meritorio" que resulta fundamental para la sociedad porque permite construir ciudadanía a través de seres humanos que tienen la capacidad de comprender el mundo en el que viven y de aportarle a la sociedad, no solo con su trabajo, sino también con sus decisiones inspiradas en un sentido ético de participar en la vida comunitaria y política. Surgen varios interrogantes respecto de cómo las inversiones en educación que alguien realiza a lo largo del tiempo inciden en su trayectoria de ingresos laborales, o hasta qué punto el bienestar de las personas está influenciado por la calidad de la educación que reciben.

Todos estos interrogantes nos llevan a proponer varios retos desde la perspectiva de lo público y lo privado. En las últimas décadas ha emergido la concepción alternativa que supone un proceso más dinámico de profesión y profesionalización, más sensible y flexible a los cambios de la situación laboral y social, que implica la posibilidad de generar conocimiento profesional especializado mediante la práctica. Se trata de un modelo de formación basado en la confianza en las capacidades de los sujetos para continuar aprendiendo y perfeccionándose, que le confiere un papel central en la posibilidad del cambio en educación. Pero también desde esta perspectiva, al asumir una profesionalidad se puede ganar en democratización del colectivo y en el desarrollo de una nueva cultura profesional que favorezca los procesos de innovación y cambio en la educación.

En el marco del proceso de reconstrucción del rol profesional, una de las tareas sustantivas por realizar es generar los espacios y tiempos necesarios para desarrollar las capacidades que le permitan observar e indagar reflexionando sobre su propia práctica: qué hacen, por qué lo hacen, qué resultados logran; reflexionar críticamente para encontrar las condiciones de modificarlas hacia formas más eficaces, democráticas y responsables. Esta es una apuesta significativa que la Universidad de La Salle tiene por los territorios rurales. Por esto viene desarrollando proyectos en educación rural con diversas instituciones educativas para el fortalecimiento de las ruralidades campesinas y étnicas, con el fin de incidir en la reformulación de política pública y de emprendimiento e innovación social y educativa mediante acciones para mejorar la calidad de la formación de maestros rurales de las escuelas normales superiores.

Finalmente, es necesario proponer una educación cualificada transdisciplinaria, situada, diferencial, inclusiva. No solo para el ser profesional, sino para el fortalecimiento de la ciudadanía desde | la perspectiva del sujeto social y político, dado que la educación en todas sus dimensiones se ofrece o postula desde su misión y visión institucional, como mediadora para la transformación de la realidad, hacia una sociedad con sustentabilidad y sostenibilidad; justicia social que logre superar las desigualdades y la inequidad. 


\section{Referencias}

Comisión Económica para América Latina y el Caribe-Cepal. (2017). Brechas, ejes y desafíos en el vínculo entre lo social y lo productivo. Santiago de Chile: onu.

Editorial: Pública la recibimos, pública la entregaremos. (2018), De la urbe, 19 (92), 2.

Decreto 316 de Remuneración Salarial, Ministerio de Educación Nacional (2018). Disponible en https://www.fecode.edu.co/images/Decretos/2018/DECRETO_SALARIAL_2018/DECRETO\%20316\%20DEL\%2019\%20FEBRERO\%20DE\%202018.pdf

Echeverri, P. R. (1998). La misión rural "Colombia en transición", de la crisis a la convivencia: una visión desde lo rural. Bogotá: ॥CA.

Editorial: Pública la recibimos, pública la entregaremos". (2018), De la urbe, 19 (92), 2.

Edu-Factory y Universidad Nómada (comps.). (2010). La universidad en conflicto: capturas y fugas en el mercado global del saber. Traficantes de Sueños. Recuperado de https://kisslibrary.net/book/E4E55499272C746208B8?utm_source=ps4\&utm_ medium $=$ disveilici.ga\&utm_campaign $=$ fnom $\& x=511123$

Las escuelas normales superiores y el Ministerio de Educación verifican las condiciones de calidad de los programas de formación complementaria. (2018). Recuperado de https://www.mineducacion.gov.co/1759/w3-article-368491.html? _noredirect=1 .

Gallego, R., Pérez, R., Torres de Gallego y Torres, L. N. (2004). Sobre la formación inicial y continua de maestros en ciencias en Colombia: un estudio a partir de programas acreditados. Ciência \& Educação, 10(2), 129-134.

Hernández, Á. M. (2018). El trasfondo de la crisis. Hacia una agenda común por las universidades públicas. [Presentación de diapositivas para el Encuentro Nacional de Profesores Universitarios. Asociación de Profesores de la Universidad de Antioquia (Asoprudea). "Crisis de la universidad pública; mitos y realidades". Universidad de Antioquia, Medellín, septiembre 18 del 2018].

Ministerio de Educación Nacional-MEN. (2013). Propuesta metodológica para la distribución de recursos artículo 87 de la Ley 30 de 1992. Vigencia 2013. Recuperado de www.mineducacion.gov.co/sistemasdeinformacion/1735/articles-212353_modelol.pdf.

Ministerio de Educación Nacional-MEN. (2016). Propuesta metodológica para la distribución de recursos artículo 87 de la ley 30 de 1992 vigencia 2016. Recuperado de www.mineducacion.gov.co/sistemasdeinformacion/1735/articles-354032_recurso_4.pdf.

Nussbaum, M. C. (2010). Sin fines de lucro. ¿̇Por qué la democracia necesita de las humanidades? Discusiones. Buenos Aires: Katz.

Pérez Gómez, A. (1995) Autonomía profesional del docente y controldemocrático". En varios autores, En P. Manzano, Volver a pensar la educación: Congreso Internacional de Didáctica (pp. 339-353). Madrid: Morata. 
Redacción Vivir. (2018, 21 de marzo). Estudiantes protestan en la Universidad Nacional por Ser Pilo Paga. El Espectador. Disponible en https://www.elespectador.com/ noticias/educacion/estudiantes-protestan-en-la-universidad-nacional-por-ser-pilo-paga-articulo-745730

Schön, D. A. (1983). El profesional reflexivo. Cómo piensan los profesionales cuando actúan. Barcelona: Paidós.

Schön, D. A. (1987), La formación de profesionales reflexivos. Hacia un nuevo diseño de la enseñanza y el aprendizaje en las profesiones. Barcelona: Paidós,

Organización de las Naciones Unidas para la Educación, la Ciencia y la Cultura-Unesco. (1996). Informe de la Comisión de Educación para el Siglo Xxı.

Organización de las Naciones Unidas para la Educación, la Ciencia y la Cultura-Unesco (1997). Educación para un futuro sostenible: una visión transdisciplinaria para una acción concertada. Paris: autor.

Organización de las Naciones Unidas para la Educación, la Ciencia y la Cultura-Unesco. (2009). La nueva dinámica de la educación superior y la investigación para el cambio social y el desarrollo. Paris: autor.

Organización de las Naciones Unidas para la Educación, la Ciencia y la Cultura-Unesco (2016). Informe de seguimiento de la educación en el mundo: la educación al servicio de los pueblos y el planeta: creación de futuros sostenibles para todos. Recuperado de https://cpalsocial.org/documentos/464.pdf

SNIES- MEN (2017). Matrícula por área de conocimiento. Disponible en https://www. mineducacion.gov.co/sistemasinfo/Informacion-a-la-mano/212400:Estadisticas

Pefil del egresado. (s.f.). En UNAD licenciatura en filosofía, Recuperado de https:// estudios.unad.edu.co/licenciatura-en-filosofia

Perfil del egresado.(s.f.). Universidad Santo Tomás, Licenciatura en Filosofía y letras. Recuperado de https://facultadfilosofiayletras.usta.edu.co/index.php/presentacion-programa-licenciatura 1

Perfil del egresado. (s.f.). UPN, Licenciatura en Química. Recuperado de http://cienciaytecnologia. pedagogica.edu.co/vercontenido. php?idp=373\&idh $=376 \&$ idn $=10118$

Zeichner, K. (1993). El maestro como profesional reflexivo. En Cuadernos de Pedagogía, 20, 44-49.

\section{Para citar este artículo}

Zapata, M. (2019). Más allá de la profesionalización: los quehaceres de la educación. Revista Tecné, Episteme y Didaxis: TED, 46, 139-156. 